\title{
The Asymptotic Estimates of Discounted Penalty Functions in a Risk Model with Random Paying Dividends to Shareholders and Policyholders
}

\author{
Lei He*, Lin Lin, Shuyi Zhou \\ Business School, Hunan Normal University, Changsha 410083, Hunan, P.R. China
}

*Corresponding Author: Lei He, Business School, Hunan Normal University, Changsha 410083, Hunan, P.R. China

\begin{abstract}
In this paper, we derive the asymptotic estimates for the Gerber-Shiu discounted penalty functions of the risk model with randomly paying dividends to shareholders and policyholders in [4] by constructing renewal equations. For the model, the asymptotic estimates for the ruin probability are obtained, which is a power function. Numerical examples show the effectiveness of the asymptotic estimates.
\end{abstract}

Keywords: Discounted penalty function, Renewal equation, Ruin probability

\section{INTRODUCTION}

We consider the surplus process

$$
U(t)=u+t-\sum_{k=1}^{t} \xi_{k} X_{k}-\sum_{k=1}^{t}\left[\eta_{k}^{(1)} I\left(U(k-1) \geq a_{1}\right)+\eta_{k}^{(2)} I\left(U(k-1) \geq a_{2}\right)\right],
$$

where $u \geq 0, a_{1} \geq 0, a_{2}>a_{1}, t \in N^{*}, \xi=\left\{\xi_{t}, t=1,2, \cdots\right\}, X=\left\{X_{t}, t=1,2, \cdots\right\}$ and $\eta^{(i)}=\left\{\eta_{t}^{(i)}, t=1,2, \cdots\right\}(i=1,2)$ are stochastic processes in some probability space $(\Omega, \mathcal{F}, P), I(B)$ is the indicator function of a set $B . \xi=\left\{\xi_{t}, t=1,2, \cdots\right\}$ is a series with the common distribution which is the binomial distribution $B(p)(0<p<1) . X=\left\{X_{t}, t=\right.$ $1,2, \cdots\}$ is assumed to be independent and identically distributed as $F=\{f(k)=\operatorname{Pr}(X=$ $k) ; k=1,2, \cdots\} . \eta^{(i)}=\left\{\eta_{t}^{(i)}, t=1,2, \cdots\right\}$ is independent and identically distributed; the common distribution is binomial distribution $B\left(q_{i}\right), q_{i} \in(0,1)(i=1,2)$, and the random series $\xi, X, \eta^{(1)}, \eta^{(2)}$ are mutually independent (see [4] for the study of related model). The model had been built to describe the fact that the joint-stock company may pay the dividends to the policyholders and shareholders by [4].

The model can be interpreted as follows: $u$ is the initial capital; $a_{1}, a_{2}$ are the thresholds of paying dividends for shareholders and policyholders respectively. The premium in unit time is one and the number of claim is $\xi_{t}$ in the time period $(t-1, t]$. If a claim occurs in $(t-1, t]$, the amount of claim is $X_{t}$. When the surplus $U(t-1)$ is not less than $a_{1}$, the dividends $\eta_{t}^{(1)}$

will be given to the policyholders. The dividends $\eta_{t}^{(2)}$ will be paid to shareholders when the

$U(t-1)$ is no less than $a_{2}$. More detail can be seen in [4].

It's known that the discounted penalty function can be used to obtain quantity related

with ruin, such as ruin probability. For discrete time risk model, recursive formulas of the discounted penalty function about initial surplus are usually calculate the quantity related with ruin. However, recursive formulas of the discounted penalty function is not effectively comparing to the asymptotic results about it. The asymptotic results about quantity related with ruin in other risk model have been studied extensively, such as [1,2,3], and so on. The estimation for the ruin probability of a discrete time model under nonnegative random interest rate has been obtained in [1]. And [3] establishes some asymptotic results for both finite and infinite ruin probability in a 
The Asymptotic Estimates of Discounted Penalty Functions in a Risk Model with Random Paying Dividends to Shareholders and Policyholders

discrete time risk model with constant interest rate and upper-tail independent for the risks which belong to regularly varying tail class. What's more, the asymptotic estimate for the discounted penalty function has been obtained in the compound binomial model with randomized decisions on paying dividends, and used to derive the asymptotic estimates for ruin probability and other quantity related with ruin in [2]. For the model (1.1), the recursive formulas of discounted penalty function has been derived (see [4]), but the asymptotic estimate for penalty function have not been obtained. Therefore, the aim of this paper is to obtain the asymptotic estimate for the discounted penalty function in [4], and use it to derive the asymptotic estimates for ruin probability and other quantities related with ruin. The results improve the study about the ruin problem under the compound binomial risk model with randomly paying dividends to shareholders and policyholders. So it is meaningful for analyzing how quantities related with ruin of joint insurance company are affected by the dividends paid to shareholders and policyholders.

This paper is organized as follows: In Section 2, the preliminaries will be shown. In

Section 3, we will derive the asymptotic estimate for the discounted penalty function. In Section 4, the asymptotic estimates of the ruin probability and the distribution function of deficit at ruin will be obtained by the discounted penalty function. The conclusions will be shown in the section 5 .

\section{Preliminaries}

Define ruin time with. $T=\operatorname{in} f\{t \geq 0 \mid U(t)<0\} \quad$ The Gerber-Shiu discounted penalty function is represented as

$$
\phi_{r}(u)=E\left[\omega\left(U_{T-1},\left|U_{T}\right|\right) I(T<+\infty) r^{T} \mid U(0)=u\right],
$$

where $\omega(x, y)$ is the non negative bounded function for $x \geq 0, y \geq 0,0<r \leq 1$. In this paper, we only obtain the asymptotic estimate for $\phi_{1}(u)$. Let $\phi(u)=\phi_{1}(u)$, i.e., the discount factor $r=1$. And the fact $\sum_{k=0}^{-1} m_{k}=0$ is adopted. For the convenient of calculation, let $P(n)=\sum_{k=1}^{n} f(k), \bar{P}(n)=1-P(n)$. we always assume $\mu=\sum_{k=1}^{+\infty} k f(k)=\sum_{k=0}^{+\infty} \bar{P}(n)<\infty$, and $E\left(\xi_{1} X_{1}+\eta_{1}^{(1)}+\eta_{1}^{(2)}\right)=p \mu+q_{1}+q_{2}<1$, which leads to a positive security loading $\theta\left(\theta=\frac{1-p \mu-q_{1}-q_{2}}{p \mu}\right)$.

Lemma 1. For the model (1.1), let

$$
\begin{array}{r}
H(x)=p_{1} p_{2} f(x)+\left(q_{1} p_{2}+p_{1} q_{2}\right) f(x-1)+q_{1} q_{2} f(x-2) \\
G(x)=p_{1} p_{2} \bar{P}(x)+\left(q_{1} p_{2}+p_{1} q_{2}\right) \bar{P}(x-1)+q_{1} q_{2} \bar{P}(x-2), \\
T(x)=p_{1} f(x)+q_{1} f(x-1), \quad L(x)=p_{1} \bar{P}(x)+q_{1} \bar{P}(x-1),
\end{array}
$$

then, (A) (i) if $a_{1}>0$, for all $u \leq a_{2}, \phi(0), \phi(1), \cdots, \phi\left(a_{2}\right)$ satisfy the following linear equations:

$$
q \phi(0)-q_{2} \phi\left(a_{2}-1\right)-q_{1} \phi\left(a_{1}-1\right)=\delta^{\prime},
$$

for $u=0,1, \cdots, a_{1}-1$,

$$
q \phi(u+1)+(p f(1)-1) \phi(u)+p \sum_{k=0}^{u-1} \phi(k) f(u+1-k)=\Delta_{1}(u=1),
$$

for $u=a_{1}, a_{1}+1, \cdots, a_{2}-1$,

$$
q p_{1} \phi(u+1)+\left(q q_{1}+p p_{1} f(1)-1\right) \phi(u)+p \sum_{k=0}^{u-1} \phi(k) T(u+1-k)=\Delta_{2}(u+1),
$$

where

$$
\Delta_{1}(u+1)=-p \sum_{k=u+2}^{+\infty} \omega(u, k-u-1) f(k)
$$


The Asymptotic Estimates of Discounted Penalty Functions in a Risk Model with Random Paying Dividends to Shareholders and Policyholders

$$
\begin{aligned}
\Delta_{2}(u+1)= & -p \sum_{k=u+1}^{+\infty} \omega(u, k-u) T(k+1) \\
\delta^{\prime}= & p \sum_{k=a_{1}}^{+\infty} \sum_{i=k}^{\infty} \omega(k, i-k+1) H(i+2)+p \sum_{k=a_{1}}^{a_{2}-2} \sum_{i=k}^{+\infty} \omega(k, i-k) f(i+1) \\
& +p p_{2} \sum_{i=a_{2}}^{+\infty} \omega\left(a_{2}-1, i-a_{2}+1\right) T(i+1) \\
& +p p_{1} \sum_{i=a_{1}}^{+\infty} \omega\left(a_{1}-1, i-a_{1}+1\right) f(i+1) .
\end{aligned}
$$

(ii) if $a_{1}=0$, for $u \leq a_{2}, \phi(0), \phi(1), \cdots, \phi\left(a_{2}\right)$ satisfy the following linear equations

$$
q p_{1} \phi(0)-q_{2} \phi\left(a_{2}-1\right)=\delta^{\prime \prime},
$$

for $u=0,1, \cdots, a_{2}-1$,

$$
a p_{1} \phi(u+1)+\left(q q_{1}+p p_{1} f(1)-1\right) \phi(u)+p \sum_{k=0}^{u-1} \phi(k) T(u+1-k)=\Delta_{2}(u+1),
$$

Where

$$
\begin{aligned}
& \delta^{\prime \prime}=p p_{2} \sum_{u=0}^{a_{2}-1} \sum_{k=u+1}^{+\infty} \omega(u, k-u) T(k+1)+p q_{2} \sum_{u=0}^{a_{2}-2} \sum_{k=u+1}^{+\infty} \omega(u, k-u) T(k+1) \\
& +p \sum_{u=a_{2}}^{+\infty} \sum_{k=u}^{+\infty} \omega(u, k-u+1) H(k+2) . \\
& \text { (B) for } u \geq a_{2}, \\
& \qquad(u+1)=\frac{1-q\left(q_{1} p_{2}+p_{1} q_{2}\right)}{q p_{2} p_{1}} \phi(u)-\frac{q_{1} q_{2}}{p_{1} p_{2}} \phi(u-1)-\frac{p}{q p_{1} p_{2}} \sum_{k=0}^{u} \phi(k) H(u+1-k) \\
& -\frac{p}{q p_{1} p_{2}} \sum_{k=u}^{+\infty} \omega(u, k-u+1) H(k+2) .
\end{aligned}
$$

Proof. See [4].

Let $D=\xi_{1} X_{1}+\eta_{1}^{(1)}+\eta_{1}^{(2)}$. Denote the generating function of $D$ by $G_{D}(r)$, then

$$
G_{D}(r)=\left(p G_{X}+q\right)\left(p_{1}+q_{1} r\right)\left(p_{2}+q_{2} r\right),
$$

where $G_{X}$ is the generating function of $X$.

Similar to the one in [2], assuming exists a $r_{\infty}$ such that $G_{X}(r) \longrightarrow+\infty\left(r \longrightarrow r_{\infty}\right)\left(r_{\infty}\right.$ is possibly $+\infty)$.

Consider equation

$$
\left(p G_{X}(r)+q\right)\left(p_{1} r+q_{1}\right)\left(p_{0}+q_{0} r\right)=r .
$$


Let $H(r)=\left(p G_{X}(r)+q\right)\left(p_{1}+q_{1} r\right)\left(p_{2}+q_{2} r\right) . H(0)=q q_{1} q_{1}, H(1)=1, H(r)$ is a convex and increasing function in $\left[0, r_{\infty}\right)$, thus equation $(2.9)$ has two real non-negative roots at most, and one of them is 1 . Because $\theta>0, H^{\prime}(1)=p \mu+q_{1}+q_{2}<1$. Owing to $H^{\prime \prime}(r)>0$ in $\left[0, r_{\infty}\right), H(r)$ is strictly convex in $\left[0, r_{\infty}\right)$. Therefore, there exist two real roots in $(2.9)$. Denote the other root by $R$, then $R>1$.

Lemma 2. $Z$ is a set of integers, $\left\{a_{k}, k \in Z\right\},\left\{b_{k}, k \in Z\right\},\left\{u_{k}, k \in Z\right\}$ are sequences that satisfy $a_{k} \geq 0, \sum_{-\infty}^{+\infty} a_{k}=1, \sum_{-\infty}^{+\infty}|k| a_{k}<+\infty, \sum_{-\infty}^{+\infty} k a_{k}>0, \sum_{-\infty}^{+\infty}\left|b_{k}\right|<+\infty$, the greatest common divisor of the integers $k$ for which $a_{k}>0$ is 1 , the bounded series $u_{k}$ satisfies the following renewal equation

$$
u_{n}=\sum_{k=-\infty}^{+\infty} a_{n-k} u_{k}+b_{n}, \quad n=0, \pm 1, \pm 2, \cdots
$$

then $\lim _{n \rightarrow \infty} u_{n}$ and $\lim _{n \rightarrow-\infty} u_{n}$ exist. Furthermore, if $\lim _{n \rightarrow-\infty} u_{n}=0$, then

$$
\lim _{n \rightarrow \infty} u_{n}=\frac{\sum_{k=-\infty}^{\infty} b_{k}}{\sum_{k=-\infty}^{\infty} k a_{k}} .
$$

Proof. See [5](Chapter 3).

\section{ASYMPTOTIC ESTIMATES}

Let

$$
\begin{aligned}
\Xi_{1}(u, \omega)= & p q_{1} \sum_{k=0}^{u-2} \sum_{i=k+2}^{+\infty} \omega(k, i-k-1) f(i)+p p_{1} \sum_{k=0}^{u-1} \sum_{i=k+2}^{+\infty} \omega(k, i-k-1) f(i), \\
\Xi_{2}(u, \omega)= & p p_{2} \sum_{k=a_{1}}^{u-1} \sum_{i=k+1}^{+\infty} \omega(k, i-k) T(i+1)+p q_{2} \sum_{k=a_{1}}^{u-2} \sum_{i=k+1}^{+\infty} \omega(k, i-k) T(i+1) \\
& +p q_{1} \sum_{k=0}^{a_{1}-2} \sum_{i=k+2}^{+\infty} \omega(k, i-k-1) f(i)+p p_{1} \sum_{k=0}^{a_{1}-1} \sum_{i=k+2}^{+\infty} \omega(k, i-k-1) f(i), \\
\Xi_{3}(u, \omega)= & p p_{2} \sum_{k=0}^{u-1} \sum_{i=k+1}^{+\infty} \omega(k, i-k) T(i+1)+p q_{2} \sum_{k=0}^{u-2} \sum_{i=k+1}^{+\infty} \omega(k, i-k) T(i+1), \\
\Xi_{4}(u, \omega)= & (R-1) R^{u} \sum_{k=u}^{+\infty} \sum_{i=k}^{+\infty} \omega(k, i-k+1) H(i+2),
\end{aligned}
$$

Theorem 1. For the model (1.1), when $a_{1}>0$, the asymptotic estimate for the discounted penalty function $\phi(u)$ is 


$$
\phi(u) \sim \frac{K_{a_{1}, a_{2}}}{K_{0}} R^{-u}
$$

Where

$$
\begin{aligned}
K_{a_{1}, a_{2}}= & K_{1}-(R-1) K_{2}-(R-1) \sum_{u=a_{1}+1}^{u_{2}} R^{u} \Xi_{2}(u, \omega)+p \sum_{u=a_{2}+1}^{+\infty} \Xi_{4}(u, \omega), \\
K_{0}= & {\left[q q_{1} q_{2}+p p_{1} p_{2} \bar{P}(1)+p\left(q_{1} p_{2}+p_{1} q_{2}\right)+p q_{1} q_{2}\right] R(R-1) } \\
& +p(R-1) \sum_{k=2}^{+\infty} k R^{k} G(k), \\
K_{1}= & (R-1)\left(q p_{1} p_{2} \phi(0)+\left(q p_{1} p_{2} \phi(1)-\left(q q_{1} q_{2}+p p_{1} p_{2} \bar{P}(1)\right) \phi(0)\right) R\right) \\
& -\left(p\left(q_{1} p_{2}+p_{1} q_{2}\right)+p q_{1} q_{2}\right) \phi(0) R(R-1)+(R-1) q p_{1} p_{2} \phi(2) R^{2} \\
& -\left(\left(\left(q q_{1} q_{2}+p p_{1} p_{2}\right) \bar{P}(1)+p\left(q_{1} p_{2} p_{1} q_{2}\right)+p q_{1} q_{2}\right) \phi(1)-p \phi(0)\right) R^{2} \\
& -q \phi(0)\left(R^{a_{2}+1}-R^{2}\right)-q \phi\left(a_{1}-1\right)\left(R^{a_{2}+1}-R^{a_{1}+1}\right), \\
K_{2}= & q_{1} p_{2} \sum_{u=2}^{a_{1}} R^{u} \phi(u-1)+q_{2} \sum_{u=2}^{a_{2}} R^{u} \phi(u-1)+q_{1} q_{2} \sum_{u=2}^{a_{1}} R^{u} \phi(u-2) \\
& +p_{2} \sum_{u=2}^{a_{1}} \Xi_{1}(u, \omega) R^{u}+p_{2} \sum_{u=2}^{a_{1}} \Xi_{1}(u, \omega) R^{u} .
\end{aligned}
$$

Proof. When $u \geq a_{2}$, rewrite (2.7), we can find

$$
\begin{aligned}
\phi(u)= & q p_{1} p_{2} \phi(u+1)+q\left(q_{1} p_{2}+p_{1} q_{2}\right) \phi(u)+q q_{1} q_{2} \phi(u-1) \\
& +p \sum_{k=0}^{u} \phi(u) H(u+1-k)+p \sum_{k=u}^{+\infty} \omega(u, k-u+1) H(k+2) .
\end{aligned}
$$

For $t \geq a_{2}$, subtracting $q \phi(u)$ from both sides of (3.2), using the $p_{1} p_{2}+q_{1} p_{2}+p_{1} q_{2}+q_{1} q_{2}=1$, summing it over $u$ from $a_{2}$ to $t$ and interchanging the order of summation, we can obtain

$$
\begin{aligned}
q p_{1} p_{2}\left(\phi(t+1)-\phi\left(a_{2}\right)\right)= & q q_{1} q_{2}\left(\phi(t)-\phi\left(a_{2}-1\right)\right)+p \sum_{k=0}^{t} \phi(u) G(t+1-k) \\
& -p \sum_{k=0}^{a_{2}-1} \phi(k) G\left(a_{2}-k\right) \\
& -p \sum_{k=a_{2}}^{t} \sum_{i=k}^{+\infty} \omega(k, i-k+1) H(i+2) .
\end{aligned}
$$

Owing to the relative security loading $\theta>0, \lim _{u \rightarrow 0} \psi=0,|\phi(u)| \leq|| \omega|| \psi(u)$, where $\|\omega\|=\sup \{\omega(x, y) \mid x \geq 0, y \geq 0\}$, then $\lim _{u \rightarrow 0} \phi(u)=0$. By the dominated convergence theorem, the following inequality can be obtained:

$$
\begin{aligned}
0 \leq p \sum_{k=0} \phi(k) G(t+1-k)= & p \sum_{k=0} \phi(t-k) G(k+1) \\
& \leq p \sum_{k=0}^{+\infty} \phi(t-k) G(k+1) \rightarrow 0 .
\end{aligned}
$$

Then, $p \sum_{k=0}^{t} \phi(k) G(t+1-k) \rightarrow 0$. Let $t \rightarrow 0$ in (3.3), it can be found that

$$
\begin{aligned}
q p_{1} p_{2} \phi\left(a_{2}\right)= & q q_{1} q_{2} \phi\left(a_{2}-1\right)+p \sum_{k=0}^{a_{2}-1} \phi(k) G\left(a_{2}-k\right) \\
& +p \sum_{k=a_{2}}^{\infty} \sum_{i=k}^{\infty} \omega(k, i-k+1) H(i+2) .
\end{aligned}
$$

(3.3) plus (3.4), we can obtain

$$
\begin{aligned}
q p_{1} p_{2} \phi(t+1)-q q_{1} q_{2} \phi(t)= & p \sum_{k=0}^{t} \phi(k) G(t+1-k) \\
& +p \sum_{k=t+1}^{+\infty} \sum_{i=k}^{+\infty} \omega(k, i-k+1) H(i+2) .
\end{aligned}
$$


Replacing $t+1$ by $t$ and plus the $\left(q_{1}+p_{1} q_{2}+p p_{1} p_{2}\right) \phi(t)$ in both sides of (3.5), we get

$$
\begin{aligned}
& \phi(t)=\left(q_{1}+p_{1} q_{2}+p p_{1} p_{2}\right) \phi(t)+p \sum_{k=t}^{+\infty} \sum_{i=k}^{+\infty} \omega(k, i-k+1) H(i+2) \\
& +\left(q q_{1} q_{2}+p p_{1} p_{2} \bar{P}(1)+p\left(q_{1} p_{2}+p_{1} q_{2}\right)+p q_{1} q_{2}\right) \phi(t-1) \\
& +p \sum_{k=0}^{t-2} \phi(k) G(t-k), t>a_{2} .
\end{aligned}
$$

Rewriting the (3.4), for $0 \leq u<a_{1}, a_{1}>0$, we can obtain

$$
\phi(u)=q \phi(u+1)+p \sum_{k=1}^{u+1} \phi(u+1-k) f(k)+p \sum_{k=u+2}^{+\infty} \omega(u, k-u-1) f(k) .
$$

Subtracting $q \phi(u)$ from both sides (3.7), summing it over $u$ from 0 to $t-1(0<t \leq$ $a_{1}$ ), and interchanging the order of summation, for $0<t \leq a_{1}$, we obtain

$$
q(\phi(t)-\phi(0))=p \sum_{k=0}^{t} \phi(k) \bar{P}(t-k)-p \sum_{u=0}^{t-1} \sum_{k=u+2}^{+\infty} \omega(u, k-u-1) f(k) .
$$

Replacing $t$ by $t-1$ in (3.8), and adding $p \phi(t-1)$ to both sides of it, for $1<t \leq a_{1}$, we get

$$
\phi(t-1)-q \phi(0)=p \sum_{k=0}^{t} \phi(k) \bar{P}(t-k)-p \sum_{u=0}^{t-2} \sum_{k=u+2}^{+\infty} \omega(u, k-u-1) f(k) \text {. }
$$

From $(3.8) \times p_{1}+(3.9) \times q_{1}$, for $1<t \leq a_{1}$ we obtain

$$
q p_{1} \phi(t)+q_{1} \phi(t-1)-q \phi(0)=p \sum_{k=0}^{t-1} \phi(k) L(t-k)-\Xi_{1}(t, \omega) .
$$

Replacing $t$ by $t-1$ in (3.10) and plus $p \phi(t-1)$, for $2<t \leq a_{1}$, we obtain

$$
\begin{aligned}
\left(q p_{1}+p\right) \phi(t-1)+q_{1} \phi(t-2)-q \phi(0)= & p \sum_{k=0}^{t-1} \phi(k) L(t-k-1) \\
& -\Xi_{1}(t-1, \omega) .
\end{aligned}
$$

From $p_{2} \times(3.10)+q_{2} \times(3.11)$, for $2<t \leq a_{1}$, we can obtain

$$
\begin{aligned}
q p_{1} p_{2} \phi(t)= & q \phi(0)-\left(q_{1} p_{2}+q p_{1} q_{2}+p q_{2}\right) \phi(t-1)-q_{1} q_{2} \phi(t-2) \\
& +p \sum_{k=0}^{t-1} \phi(k) G(t-k)-p_{2} \Xi_{1}(t, \omega)-q_{2} \Xi_{1}(t-1, \omega) .
\end{aligned}
$$

Rewriting (2.4), for $a_{1} \leq u<a_{2}$, we obtain

$$
\phi(u)=q p_{1} \phi(u)+p \sum_{k=1}^{u} \phi(k) T(u+1-k)+p \sum_{u+1}^{+\infty} \omega(u, k-u) T(k+1) .
$$

Subtracting $q \phi(u)$ from both sides of (3.13), summing it over $u$ from $a_{1}$ to $t-1\left(a_{1} \leq t<a_{2}\right)$ and interchanging the order of summation, we obtain

$$
q p_{1}\left(\phi(t)-\phi\left(a_{1}\right)\right)=p \sum_{k=0}^{t-1} \phi(k) L(t-k)-p \sum_{k=0}^{t-1} \phi(k) L\left(a_{1}-k\right)
$$




$$
-p \sum_{u=a_{1}}^{t-1} \sum_{k=u+1}^{+\infty} \omega(u, k-u) T(k+1) .
$$

Let $t=a_{1}$ in (3.10), we can obtain

$$
q p_{1} \phi\left(a_{1}\right)+q_{1} \phi\left(a_{1}-1\right)-q \phi(0)=p \sum_{k=0}^{a_{1}-1} \phi(k) L(t-k)-\Xi_{1}(t, \omega) \text {. }
$$

Adding (3.14) to (3.15), for $a_{1} \leq t<a_{2}$, we obtain

$$
\begin{aligned}
q p_{1} \phi(t)+q_{1} \phi\left(a_{1}-1\right)-q \phi(0)= & p \sum_{k=0}^{t-1} \phi(k) L(t-k) \\
& -p \sum_{u=a_{1}}^{t-1} \sum_{k=u+1}^{+\infty} \omega(u, k-u) T(k+1) .
\end{aligned}
$$

Replacing $t$ by $t-1$ in the (3.16), and adding $p \phi(t-1)$ to both sides of it, for $a_{1}<t \leq a_{2}$, we can obtain

$$
\begin{aligned}
\left(p+q p_{1}\right) \phi(t-1)+q_{1} \phi\left(a_{1}-1\right)= & q \phi(0)+p \sum_{k=0}^{t-1} \phi(k) L(t-k-1) \\
& -p \sum_{u=a_{1}}^{t-2} \sum_{k=u+1}^{+\infty} \omega(u, k-u) T(k+1) .
\end{aligned}
$$

From $p_{2} \times(3.16)+q_{2} \times(3.17)$, and plus $\left(q_{1}+p_{1} q_{2}+p p_{1} p_{2}\right) \phi(t)$ in both sides of it, for $a_{1}<t \leq a_{2}$, we obtain

$$
\begin{aligned}
\phi(t)= & \left(q_{1}+p_{1} q_{2}+p p_{1} p_{2}\right) \phi(t)-\Xi_{2}(t, \omega)-q_{2} \phi(t-1)-q_{1} \phi\left(a_{1}-1\right)+q \phi(0) \\
& +\left(q q_{1} q_{2}+p p_{1} p_{2} \bar{P}(1)+p\left(q_{1} p_{2}+p_{1} q_{2}\right)+p q_{1} q_{2}\right) \phi(t-1) \\
& +p \sum_{k=0}^{t-2} \phi(k) G(t-k) .
\end{aligned}
$$

Combining (3.12), (3.18) and (3.6), we get

$$
\begin{aligned}
\phi(u)= & \left(q_{1}+p_{1} q_{2}+p p_{1} p_{2}\right) \phi(u) \\
& +\left(q q_{1} q_{2}+p p_{1} p_{2} \bar{P}(1)+p\left(q_{1} p_{2}+p_{1} q_{2}\right)+p q_{1} q_{2}\right) \phi(u-1) \\
& +p \sum_{k=0}^{u-2} \phi(k) G(u-k)
\end{aligned}
$$$$
+ \begin{cases}q p_{1} p_{2} \phi(1)-\left(q q_{1} q_{2}+p p_{1} p_{2} \bar{P}(1)\right. & u=1, \\ \left.+p\left(q_{1} p_{2}+p_{1} q_{2}\right)+p q_{1} q_{2}\right) \phi(0), & \\ q p_{1} p_{2} \phi(2)-\left(q q_{1} q_{2}+p p_{1} p_{2} \bar{P}(1)\right) \phi(1) & u=2, \\ -\left(p\left(q_{1} p_{2}+p_{1} q_{2}\right)+p q_{1} q_{2}\right) \phi(1)-p \phi(0), & \\ q \phi(0)-\left(q_{1} p_{2}+q q_{2}\right) \phi(u-1)-q_{1} q_{2} \phi(u-2) & 2<u \leq a_{1}, \\ -p_{2} \Xi_{1}(u, \omega)-q_{2} \Xi_{1}(u-1, \omega), & a_{1}<u \leq a_{2}, \\ q \phi(0)-q q_{2} \phi(u-1)-q \phi\left(a_{1}-1\right)-\Xi_{2}(u, \omega), & a_{2}<u . \\ p \sum_{k=u}^{+\infty} \sum_{i=k}^{+\infty} f(k, i-k) H(i+2), & \end{cases}
$$ 
We denote $\bar{\phi}(u)=\phi(u) R^{u}$,

$$
a_{u}= \begin{cases}q_{1}+p_{1} q_{2}+p p_{1} p_{2}, & u=0 \\ R\left(q q_{1} q_{2}+p p_{1} p_{2} \bar{P}(1)+p\left(q_{1} p_{2}+p_{1} q_{2}\right)+p q_{1} q_{2}\right), & u=1 \\ p R^{u} G(u), & u \geq 2\end{cases}
$$

$$
b_{u}= \begin{cases}q p_{1} p_{2}, & u=0, \\ R\left(q p_{1} p_{2} \phi(1)-\left(q q_{1} q_{2}+p p_{1} p_{2} \bar{P}(1),\right.\right. & u=1, \\ \left.\left.+p\left(q_{1} p_{2}+p_{1} q_{2}\right)+p q_{1} q_{2}\right) \phi(0)\right), & \\ R^{2}\left(q p_{1} p_{2} \phi(2)-\left(q q_{1} q_{2}+p p_{1} p_{2} \bar{P}(1)\right)\right) & u=2, \\ -R^{2}\left(\left(p\left(q_{1} p_{2}+p_{1} q_{2}\right)+p q_{1} q_{2}\right) \phi(1)-p \phi(0)\right), & \\ R^{u}\left(q \phi(0)-\left(q_{1} p_{2}+q_{2}\right) \phi(u-1)-q_{1} q_{2} \phi(u-2)\right) & \\ -R^{u}\left(p_{2} \Xi_{1}(u, \omega)+q_{2} \Xi_{1}(u-1, \omega)\right), & 2<u \leq a_{1}, \\ R^{u}\left(q \phi(0)-q_{2} \phi(u-1)-q \phi\left(a_{1}-1\right)-\Xi_{2}(u, \omega)\right), & a_{1}<u \leq a_{2}, \\ R^{u} p \sum_{k=u}^{+\infty} \sum_{i=k}^{+\infty} f(k, i-k) H(i+2), & a_{2}<u .\end{cases}
$$

Multiplying (3.19) by $R^{u}$, we can obtain

$$
\bar{\phi}(u)=\sum_{k=0}^{u} a_{n-k} \bar{\phi}(k)+b_{u}, \quad u=0,1,2, \cdots \text {. }
$$

We will prove that the (3.20) satisfies the conditions of Lemma 2.

$$
\begin{aligned}
\sum_{k=0}^{+\infty} a_{k}= & q_{1}+p_{1} q_{2}+p p_{1} p_{2}+R\left(q q_{1} q_{2}+p p_{1} p_{2} \bar{P}(1)+p\left(q_{1} p_{2}+p_{1} q_{2}\right)+p q_{1} q_{2}\right) \\
& +\sum_{k=2}^{+\infty} p G(k) R^{k}+p q_{1} q_{2} R^{2} \sum_{k=0}^{+\infty} \bar{P}(k) R^{k} \\
= & q_{1}+p_{1} q_{2}+q_{1} q_{2} R+\left(p p_{1} p_{2}+p\left(q_{1} p_{2}+p_{1} q_{2}\right) R+p q_{1} q_{2} R^{2}\right) \frac{G_{X}(R)-1}{R-1} \\
= & 1 .
\end{aligned}
$$

where the last equation is valid because $R$ is the root of the (2.9). The following step is to prove $\sum_{k=0}^{+\infty}\left|b_{k}\right|<\infty$. For $u>x$

$$
0 \leq b_{u} \leq p\|\omega\| R^{u} \sum_{k=u}^{+\infty} \bar{J}(k-1) G(k+1),
$$

then,

$$
\sum_{u=a_{2}+1}^{+\infty} b_{u} \leq p\|\omega\| \sum_{u=1}^{+\infty} R^{u} \sum_{k=u}^{+\infty} G(k+1) .
$$

Considering the right part of the above inequality

$$
p \sum_{u=1}^{+\infty} R^{u} \sum_{k=u}^{+\infty} G(k+1)=p \sum_{k=1}^{+\infty} \sum_{u=1}^{k} R^{u} G(k+1)
$$




$$
\begin{aligned}
& =p \sum_{k=1}^{+\infty} G(k+1) \frac{R^{k}-R}{R-1} \\
& \leq \frac{1}{R(R-1)} \sum_{k=1}^{+\infty} p G(k+1) R^{k+1} \\
& \leq \frac{1}{R(R-1)} \sum_{k=0}^{+\infty} a_{k} \\
& =\frac{1}{R(R-1)} .
\end{aligned}
$$

From (3.21) and (3.22), we can obtain $\sum_{u=a_{2}+1}^{+\infty} b_{u}<+\infty$. And Because $\left|b_{k}\right|<+\infty(0 \leq u \leq$ $\left.a_{2}\right), \sum_{u=0}^{+\infty} b_{u}<+\infty$.

$$
\sum_{u=0}^{+\infty} b_{u}=\frac{K_{1}}{R-1}-K_{2}-\sum_{u=a_{1}+1}^{a_{2}} R^{u} \Xi_{2}(u, \omega)+p \sum_{u=a_{2}+1}^{+\infty} \Xi_{4}(u, \omega) .
$$

According to Lemma 2.2, we can get

$$
\lim _{u \rightarrow+\infty} \bar{\phi}(u)=\frac{K_{a_{1}, a_{2}}}{K_{0}} .
$$

(3.24) is equivalent to (3.1). The proof is completed.

Theorem 2. For the model (1.1), when $a_{1}=0$, the asymptotic estimate for the discounted penalty function $\phi(u)$ is

$$
\phi(u) \sim \frac{K\left(0, a_{2}\right)}{K_{0}^{\prime}} R^{-u}
$$

where

$$
\begin{gathered}
K\left(0, a_{2}\right)=K_{3}-q_{2}(R-1) \sum_{u=1}^{a_{2}} R^{u} \phi(u-1)-(R-1) \sum_{u=1}^{a_{2}} R^{u} \Xi_{3}(u, \omega) \\
+p(R-1) \sum_{u=a_{2}}^{+\infty} \Xi_{4}(u, \omega) \\
K_{0}^{\prime}=\left[q q_{1} q_{2}+p p_{1} p_{2} \bar{P}(1)+p\left(q_{1} p_{2}+p_{1} q_{2}\right)+p q_{1} q_{2}\right] R(R-1)+p(R-1) \sum_{k=2}^{+\infty} k R^{k} G(k), \\
K_{3}=\begin{array}{l}
q p_{1} p_{2} \phi(0)(R-1)+q p_{1} \phi(0)\left(R^{u}-R\right) \\
+\left(q p_{1} p_{2} \phi(1)-\left(q q_{1} q_{2}+p p_{1} p_{2} \bar{P}(1)+p\left(q_{1} p_{2}+p_{1} q_{2}\right)+p q_{1} q_{2}\right) \phi(0)\right) R(R-1) .
\end{array}
\end{gathered}
$$


Proof. Subtracting $q \phi(u)$ from both sides of (2.6), summing it over $u$ from 0 to $t-1(0<t \leq$ $a_{2}$ ) and interchanging the order of summation, we obtain

$$
q p_{1}(\phi(t)-\phi(0))=p \sum_{k=0}^{t-1} \phi(k) L(t-k)-p \sum_{u=0}^{t-1} \sum_{t=u+1}^{+\infty} \omega(u, k-u) T(k+1) .
$$

Replacing $t$ by $t-1$ in (3.26) and adding $p \phi(t-1)$ to both sides of it, for $1<t \leq a_{2}$, we get

$$
\begin{aligned}
\left(q p_{1}+p\right) \phi(t-1)-q p_{1} \phi(0)= & p \sum_{k=0}^{t-1} \phi(k) L(t-k-1) \\
& -p \sum_{u=0}^{t-2} \sum_{k=u+1}^{+\infty} \omega(u, k-u) T(k+1) .
\end{aligned}
$$

From $(3.26) \times p_{2}+(3.27) \times q_{2}$, for $1<t \leq a_{2}$, and plus $\left(q_{1}+p_{1} q_{2}+p p_{1} p_{2}\right) \phi(u)$ in both sides of it,we obtain

$$
\begin{aligned}
\phi(u)= & \left.\left(q_{1}+p_{1} q_{2}+p p_{1} p_{2}\right) \phi(u)\right)+p \sum_{k=0}^{u-2} \phi(k) G(u-k) \\
& +\left(q q_{1} q_{2}+p p_{1} p_{2} \bar{P}(1)+p\left(q_{1} p_{2}+p_{1} q_{2}\right)+p q_{1} q_{2}\right) \phi(u-1) \\
& +q p_{1} \phi(0)-q_{2} \phi(u-1)-\Xi_{3}(u, \omega), 1<t \leq a_{2} .
\end{aligned}
$$

Combining the (3.28) and (3.6), we can obtain

$$
\begin{aligned}
\phi(u)= & \left(q_{1}+p_{1} q_{2}+p p_{1} p_{2}\right) \phi(u) \\
& +\left(q q_{1} q_{2}+p p_{1} p_{2} \bar{P}(1)+p\left(q_{1} p_{2}+p_{1} q_{2}\right)+p q_{1} q_{2}\right) \phi(u-1) \\
& +p \sum_{k=0}^{u-2} \phi(k) G(u-k) \\
& + \begin{cases}q p_{1} p_{2} \phi(1)-\left(q q_{1} q_{2}+p p_{1} p_{2} \bar{P}(1)\right) \phi(0) \\
-\left(p\left(q_{1} p_{2}+p_{1} q_{2}\right)+p q_{1} q_{2}\right) \phi(0), & u=1 \\
q p_{1} \phi(0)-q_{2} \phi(u-1)-\Xi_{3}(u, \omega), & 0<u \leq a_{2}, \\
p \sum_{k=u}^{+\infty} \sum_{i=k}^{+\infty} f(k, i-k) H(i+2), & a_{2}<u .\end{cases}
\end{aligned}
$$

We denote $\bar{\phi}(u)=\phi(u) R^{u}$

$$
\begin{aligned}
& a_{u}= \begin{cases}q_{1}+p_{1} q_{2}+p p_{1} p_{2}, & u=0, \\
R\left(q q_{1} q_{2}+p p_{1} p_{2} \bar{P}(1)+p\left(q_{1} p_{2}+p_{1} q_{2}\right)+p q_{1} q_{2}\right), & u=1, \\
p R^{u} G(u), & u \geq 2 .\end{cases} \\
& b_{u}= \begin{cases}q p_{1} p_{2}, & u=0, \\
\left(q p_{1} p_{2} \phi(1)-\left(q q_{1} q_{2}+p p_{1} p_{2} \bar{P}(1)+p\left(q_{1} p_{2}+p_{1} q_{2}\right)\right) \phi(0)\right) R & \\
+p q_{1} q_{2} \phi(0) R, & u=1, \\
\left(q p_{1} \phi(0)-q_{2} \phi(u-1)-\Xi_{3}(u, \omega)\right) R^{u}, & 1<u \leq a_{2}, \\
p \Xi_{4}(u, \omega), & a_{2} \leq u .\end{cases}
\end{aligned}
$$


Similarly to Theorem 3.1 , we also can prove $\sum a_{k}=1$, and $\sum\left|b_{k}\right|<+\infty$. According to the lemma 2.2 , we can obtain

$$
\lim _{u \rightarrow+\infty} \bar{\phi}(u)=\frac{K\left(0, a_{2}\right)}{K_{0}^{\prime}} .
$$

(3.30) is equivalent to (3.25). The proof is completed

\section{The Application of the Penalty Function}

We will give some examples of ruin quantities (such as the ultimate ruin probability) to illustrate the application of the recursive formulas and asymptotic estimates for the penalty function $\phi(u)$.

Example 1. Let $\omega(x, y)=1, \phi(u)=\operatorname{Pr}(T<+\infty \mid U(0)=u)=\psi(u)$, which is the ultimate ruin probability. By Theorem 3.1, when $a_{1}>0$, the asymptotic estimate for $\psi(u)$ is

$$
\psi(u) \sim \frac{K_{a_{1}, a_{2}}^{\psi}}{K_{0}} R^{-u},
$$

where

$$
\begin{aligned}
& K_{a_{1}, a_{2}}^{\psi}=K_{1}-(R-1) K_{2}-(R-1) \sum_{u=a_{1}+1}^{a_{2}} R^{u} \Xi_{2}(u, 1)+p(R-1) \sum_{u=a_{2}+1}^{+\infty} \Xi_{4}(u, 1) \\
& \Xi_{1}(u, 1)=p p_{1} \bar{P}(u)+p \sum_{k=0}^{u=0} \bar{P}(k+1) \\
& \Xi_{2}(u, 1)=p p_{2} \bar{T}(u)+p p_{1} \bar{P}(u)+p \sum_{k=0}^{u-2} \bar{T}(k+1)+p \sum_{k=0}^{a_{2}-2} \bar{P}(k+1), \\
& K_{1}=(R-1)\left(q p_{1} p_{2} \psi(0)+\left(q p_{1} p_{2} \psi(1)-\left(q q_{1} q_{2}+p p_{1} p_{2}\right) \bar{P}(1)\right) \psi(0) R\right) \\
& \left.+\left(p\left(q_{1} p_{2}+p_{1} q_{2}\right)+p q_{1} q_{2}\right) \psi(0) R(R-1)+(R-1) R^{2}\left(q p_{1} p_{2} \psi(2)\right)\right) \\
& +(R-1)\left(\left(\left(q q_{1} q_{2}+p p_{1} p_{2}\right) \bar{P}(1)+p\left(q_{1} p_{2} p_{1} q_{2}\right)+p q_{1} q_{2}\right) \psi(1)\right) R^{2} \\
& -(R-1) R^{2} p \psi(0)-q \psi(0)\left(R^{a_{2}+1}-R^{2}\right)-q \psi\left(a_{1}-1\right)\left(R^{a_{2}+1}-R^{a_{1}+1}\right), \\
& K_{2}=q_{1} p_{2} \sum_{u=2}^{a_{1}} R^{u} \psi(u-1)+q_{2} \sum_{u=2}^{a_{2}} R^{u} \psi(u-1)+q_{1} q_{2} \sum_{u=2}^{a_{1}} R^{u} \psi(u-2) \\
& +p_{2} \sum_{u=2}^{a_{1}} \Xi_{1}(u, 1) R^{u}+q_{2} \sum_{u=2}^{a_{1}} \Xi_{1}(u-1,1) R^{u} \\
& \Xi_{4}(u, 1)=R^{u} \sum_{k=u+1}^{+\infty} G(k) . \\
& +p_{2} \sum_{u=2}^{a_{1}} \Xi_{1}(u, 1) R^{u}+q_{2} \sum_{u=2}^{a_{1}} \Xi_{1}(u-1,1) R^{u}, \\
& \Xi_{4}(u, 1)=R^{u} \sum_{k=u+1}^{+\infty} G(k)
\end{aligned}
$$


The Asymptotic Estimates of Discounted Penalty Functions in a Risk Model with Random Paying Dividends to Shareholders and Policyholders

Table1. Adjustment coefficients

\begin{tabular}{|l|c|c|c|c|}
\hline$Q$ & $(0.015,0)$ & $(0.015,0.025)$ & $(0.015,0.055)$ & $(0.035,0.055)$ \\
\hline$R$ & 1.04417 & 1.041497 & 1.03812 & 1.035755 \\
\hline
\end{tabular}

\section{NUMERICAL SCHEME}

In [4], the initial term $\phi(0), \phi(1), \cdots, \phi\left(a_{2}\right)$ can be obtained by solving the set of linear equations, and $\phi\left(a_{2}+1\right), \phi\left(a_{2}+2\right)$, are deduced by the recursive formulas in Lemma 2.1. We will compare the asymptotic values for the ruin probability, and analysis on the impact of the randomly paying dividends on the ruin probability.

The numerical analysis will be carried out under assumption of parameter as follow- ing: the distribution of claim amount $X_{i}$ is a zero-truncated geometric distribution with parameter $\alpha=7 / 8$, then $f(k)=(1-7 / 8)(1 / 8)^{i-1}, i=1,2, \cdots ; p=0.85$, the threshold $\left(a_{1}, a_{2}\right)=(3,5)$. And four cases with the probability of paying dividend $Q=\left(q_{1}, q_{2}\right)=$ $(0.015,0),(0.015,0.025),(0.015,0.055),(0.035,0.055)$ will be performed. Under the four cases, the relative security loading $\theta>0$, thus the set of linear equations has unique solution in Lemma 2.1, and the adjustment coefficient $R$ exist. The adjustment coefficient $R$ are

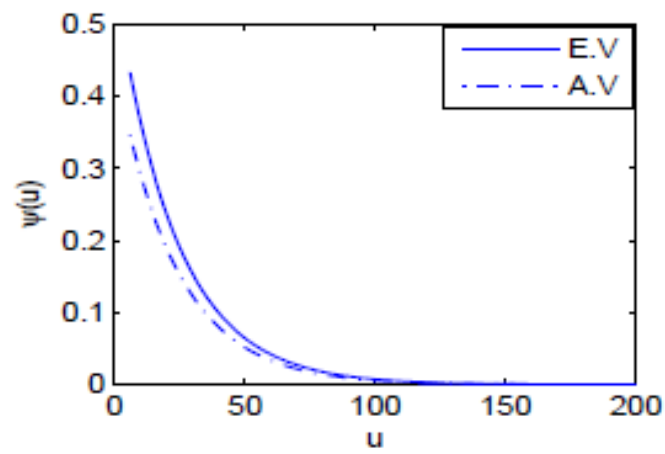

(a) $Q=(0.015,0)$

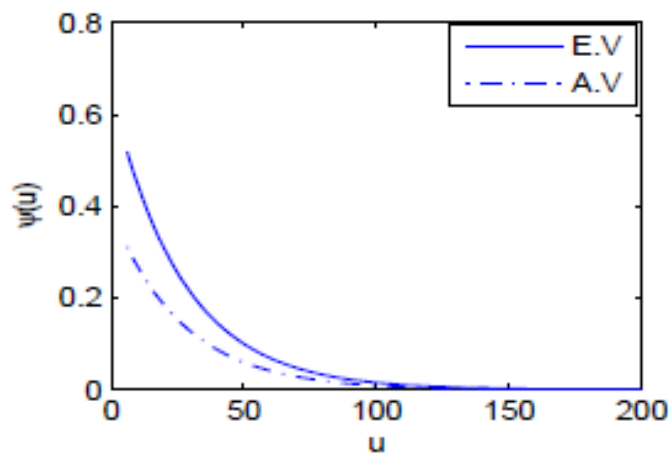

(c) $Q=(0.015,0.055)$

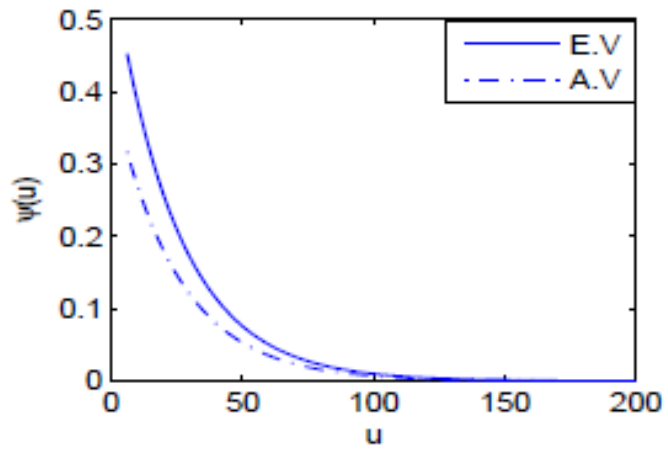

(b) $Q=(0.015,0.025)$

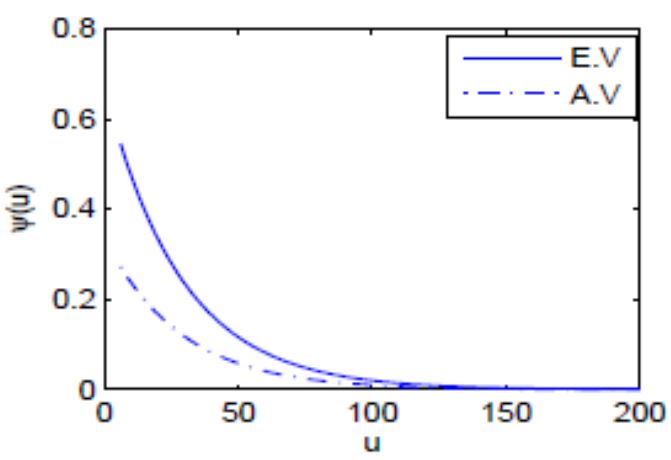

(d) $Q=(0.035,0.055)$

Figure1. Exact values vs asymptotic values for the ruin probability

computed and shown in Table 1. The exact values that calculated by recursive formulas in lemma 2.1(see[4]) and the asymptotic values that are estimated by (3.1) are shown in 1 for the ruin probability and the distribution of the deficit at ruin. In all of figures, the E.V means the exact value, and the A.V means the asymptotic value.

Figure 1 shows that compare exact values with asymptotic values for the ruin probability under the four cases with different probability of paying dividends. From the Figure 1, we can find the asymptotic values are constantly close to the exact values with the surplus $u$ increasing, and are equal to the exact values when the $u$ is large enough.

\section{CONCLuSion}

The asymptotic estimate for the Gerber-Shiu discounted penalty function of the com- pound binomial risk model with randomly paying dividends to shareholders and policyhold- ers in[4] 
The Asymptotic Estimates of Discounted Penalty Functions in a Risk Model with Random Paying Dividends to Shareholders and Policyholders

can be calculated in two case that the threshold of paying dividends to shareholders $a_{1}=0$ and $a_{1}$ $>0$. And the asymptotic estimates of the ruin probability and the distribution function of deficit at ruin have been derived by the asymptotic estimate for the discounted penalty function in the case $a_{1}=0$ and $a_{1}>0$.

\section{ACKNOWLEDGEMENT}

The project supported by National Natural Science Foundation of China (71501070) and Education Department foundation of Hunan Province (15B150).

\section{REFERENCES}

[1] Shen, X.M., Lin, Z.Y., Zhang, Y., Uniform estimate for maximum of randomly weighted sums with applications to ruin theory, Methodology. Computing in Appllied. Probabil- ity,2009, 11(2): 669-685.

[2] Tan, J., Yang, X.Q., The compound binomial model with randomized decisions on paying dividends.Insurance: Mathematics and Economics,2006, 39(1), 1-18.

[3] Weng, C.,Zhang, Y., Tan, K.S, Ruin probabilities in a discrete time risk model with dependent risks of heavy tail, Scandinavian Actuarial Journal, 2009, 3(4): 205-218.

[4] He, L., Yang, X.Q., The compound binomial model with randomly paying dividend- s to shareholders and policyholders,Insurance: Mathematics and Economics, 2010, 46(3):443-449.

[5] Kalin,S., Taylor, H.M., A first course in stochastic processes, Academic Press, New York, 1975.

[6] Cheng, S., Gerber, H.U., Shiu, E.S.W.:Discounted probabilities and ruin theory in the compound binomial model, Insurance: Mathematics and Economics, 2000, 26(2-3): 239-250.

[7] Cossette, H., Landriault, D., Marceau, E.:Ruin probabilities in the compound Markov binomial model, Scandinavian Actuarial Journal,2003,4(1): 301-323.

[8] Dickson, D.C.M.:Some comments on the compound binomial model, ASTIN Bul- letin,1994,24(1): 33-45.

[9] Gerber, H.U.:Mathematical fun with the compound Poisson process, ASTIN Bulletin, 1998, 18(2):161168.

[10] Grandell, J., Aspects of Risk Theory, Springer, New York, 1993.

[11] Shiu, E.S.W., The probability of eventual ruin in compound binomial model, ASTIN Bulletin, 1989, 19(2)(1989): 179-190.

[12] Xiao, Y.T., Guo, J.Y., The compound binomial risk model with time-correlated claims, Insurance: Mathematics and Economics, 2007, 41(1): 124-133.

[13] Liu,C., Zhang Z.,A discrete risk model with delayed claims and randomized dividend strategy,Advances in Difference Equations,2015, 12(1): 1-14.

Citation: Lei He, et.al., (2019). The Asymptotic Estimates of Discounted Penalty Functions in a Risk Model with Random Paying Dividends to Shareholders and Policyholders. International Journal of Scientific and Innovative Mathematical Research (IJSIMR), 7(10), pp. 21-33. http://dx.doi.org/ 10.20431/2347 3142.0710003

Copyright: () 2019 Authors, this is an open-access article distributed under the terms of the Creative Commons Attribution License, which permits unrestricted use, distribution, and reproduction in any medium, provided the original author and source are credited. 